\title{
A $10 \mathrm{Gbit} / \mathrm{s}, 160 \mathrm{Gchip} / \mathrm{s}$ OCDMA coding:decoding system based on superstructured fiber gratings
}

\author{
P.C. Teh, P. Petropoulos, M. Ibsen, D.J. Richardson \\ Optoelectronics Research Centre \\ Southampton University \\ United Kingdom \\ pp@orc.soton.ac.uk
}

The area of Optical Code Division Multiple Access (OCDMA) systems has received steadily growing interest in recent years [1-8]. OCDMA offers a number of attractive features for future local area networks including higher connectivity, asynchronous multiple-access and more flexible bandwidth management. Much of the increased activity in this area has resulted from improvements in Fiber Bragg Grating (FBG) fabrication technology driven mainly by the stringent requirements of DWDM. It is now possible to design and reliably fabricate superstructured FBGs with truly complex amplitude and phase responses [9], opening up the possibility of using FBG components to perform fundamental OCDMA functions such as the coding and decoding of chip patterns described herein. Superstructured gratings offer advantages in terms of compactness, scalability, integrability, cost and ease of manufacture relative to competing technological approaches. These alternative approaches include the use of: arrayed waveguide gratings [2], fiber delay line arrays [3], arrays of discrete FBGs [4], and bulk-grating based systems incorporating some form of spatial light modulator [5].

In earlier work we demonstrated the generation of seven-chip, direct sequence, unipolar code OCDMA bits at $125 \mathrm{MHz}$ using a superstructured FBG, and demonstrated optical pattern recognition using a matched FBG filter as a decoder [7]. The general approach has been extended more recently by other authors to bipolar (phase) encoding, resulting in improved code correlation signatures [8]. The repetition rate in these more recent experiments was $20 \mathrm{MHz}$ with a chip duration of $\sim 30 \mathrm{ps}$. The FBGs used were of a segmented composite form written through a specially patterned phase mask and had a reflectivity of just $1 \%$.

In this paper we present results on upgrading the superstructure grating approach to both far higher data rates (10 Gbit/s), far shorter chip-lengths (6.4ps) and far higher grating reflectivities (up to 50\%) than previously demonstrated. We demonstrate the flexibility and precision of our continuous scanning fiber/phase mask technique by fabricating both unipolar and bipolar coding and decoding gratings with close to the theoretically designed spectral response, and time domain performance characteristics. We present the results of BER measurements at $10 \mathrm{Gbit} / \mathrm{s}$ on a decoded pulse sequence both before and after transmission through $25 \mathrm{~km}$ of standard fiber which show there to be no noise-penalty associated with either the codingdecoding process, or the transmission of the coded pulse itself.

Our experimental set-up is shown in Fig. 1 and comprises a $10 \mathrm{Gbit} / \mathrm{s}, \sim 2$ ps pulse transmitter (based on a regeneratively mode-locked, soliton fiber ring laser operating at $10 \mathrm{GHz}$ ), coding and decoding gratings, and an (optional) $25 \mathrm{~km}$ standard fiber transmission span which had its dispersion compensated with a chirped FBG.

We fabricated a matched pair of both unipolar and bipolar seven-chip M-sequence, coding/decoding gratings. The unipolar gratings were similar in design to those used in our earlier experiments [7], only physically much shorter in length. The total grating length in each instance was $4.64 \mathrm{~mm}$ (corresponding to a temporal code length of $44.8 \mathrm{ps}$ ) and the individual chip width was $0.66 \mathrm{~mm}$ (corresponding to a chip length of $6.4 \mathrm{ps}$ ). Note that grating phase control can be maintained over lengths of order $10 \mathrm{~cm}$ allowing for considerably longer code sequences than demonstrated herein. The amplitude-modulated superstructure profile 
used to write the unipolar code grating is shown inset in Fig.2a along with the corresponding theoretical and experimental power reflectivity profiles. The bipolar grating design is shown inset in Fig.2b, and is a pure phase-encoded structure with discrete $\pi$ phase shifts at the (NRZ) chip transition boundaries. The experimental and theoretical plots are shown in Fig.2b. The agreement between the theoretical and experimental spectral responses of both FBG types is seen to be excellent, highlighting the precision of our grating writing process. Note that all of the gratings used in these experiments (including the dispersion compensating FBG) were written by appropriate UV exposure through the same, uniform period phase mask. The decode gratings in both instances are essentially identical to the encoder gratings other than that they have a spatially-reversed refractive index superstructure.

Since our gratings are still relatively weak we are in the Fourier theory grating design limit, such that the impulse response of the grating in the time domain is essentially given by the superstructure modulation profile used to write the grating. We examined the intensity autocorrelation functions of the incident 2 ps pulses on reflection from the individual coding/decoding gratings, and found the profiles to be in excellent agreement with our theoretical predictions. in Fig. 3 we plot the experimental and theoretical intensity autocorrelations functions of the decoder response to the incident code patterns for both the unipolar and polar cases respectively. The agreement is seen to be excellent in both cases. The system benefits of using the bipolar gratings due to the interferometric background cancellation are self-evident. In Fig.4 we plot the decoder response to the code after it has propagated over the $25 \mathrm{~km}$ dispersion-compensated transmission line, there is evidence of some correlation signal degradation, however the effects are slight, and in fact negligible in terms of overall system performance.

We performed BER measurements on the coding:decoding process for the bipolar grating, both with and without the $25 \mathrm{~km}$ transmission. The results are summarised in Fig. 5 where it is seen that no power penalty associated with code-decode process is observed in either instance. Eye diagrams for both the simple code:decode and transmitted code:decode case are shown inset within Fig.5. As expected no evidence of temporal features away from the main, chip-length long, correlation peak is observed.

In conclusion we have demonstrated high-quality, $10 \mathrm{Gbit} / \mathrm{s}$ unipolar and bipolar OCDMA coding and decoding using superstructured FBGs. The code chip rate was $160 \mathrm{Gchip} / \mathrm{s}$. Errorfree operation with no power penalty was obtained for the coding:decoding process after propagation of the code through $25 \mathrm{~km}$ of fiber. Our results highlight the precision and flexibility of our particular grating writing process and point to further applications of superstructured FBGs in future high-speed optical networks. For example our results demonstrate the possibility of using FBGs for header recognition in $160 \mathrm{Gbit} / \mathrm{s}$ OTDM based networks.

The authors wish to acknowledge useful past discussions and input from A.Fu, H.Geiger, M.N. Zervas and R.I. Laming.

\section{References}

[1] N. Karafolas Opt. Fiber Teclmol., 2, pp149-168, (1996)

[2] H.Tsuda et al.: Electron. Letts., 35, pp1187-1188, (1999)

[3] N. Wada et al.: IEEE J. Lightwave Technology, 17, pp1758-1765, (1999)

[4] H. Fathallah et al.: IEEE J. Lightwave Technology, 17, pp397-405, (1999)

[5] J. Salehi et al. IEEE J. Lightwave Technology, 17, pp478-491, (1990)

[6] L.R. Chen et al. Applied Optics, 38, pp4500-4508, (1999)

[7] H.Geiger et al.: Proc. ECOC'98, Vol.1, pp337-338 (1998)

[8] A.Grunnet-Jepsen et al.: IEEE Photon Technol. Letts., 11, 1283-1285, (1999)

[9] M. Ibsen et al: IEEE Phot. Tech. Lett. 10, 842 (1998) 


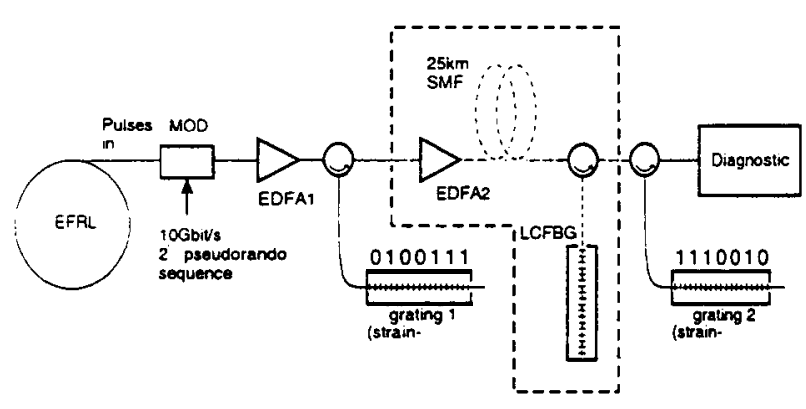

Fig. 1 Experimental set-up. The pseudorandom sequence is $2^{31}-1$ bits long. LCFBG: Linearly chirped fibre Bragg grating

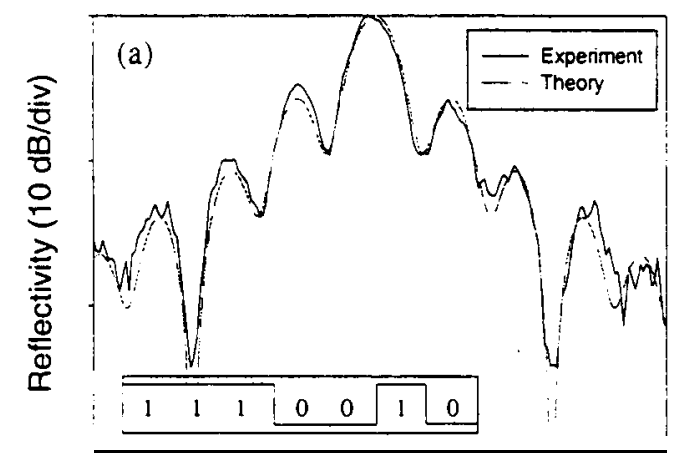

1555.61556 .01556 .41556 .81557 .2

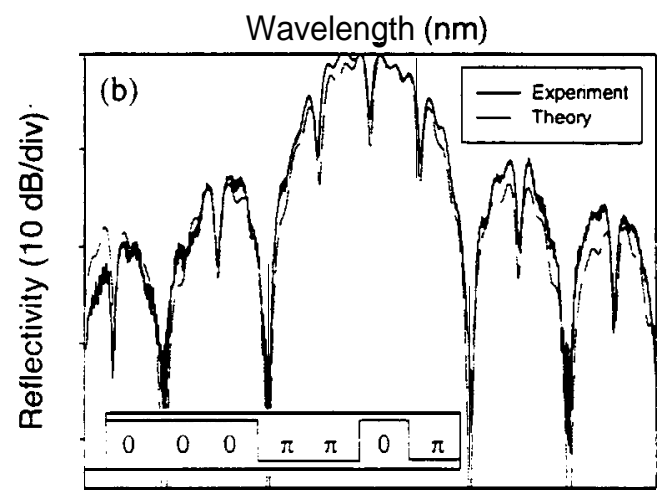

15531554155515561557155815591560

Wavelength $(\mathrm{nm})$

Fig.2 (a) Unipolar grating reflectivity spectrum (theoretical and experimental). The refractive index amplitude superstructure is shown inset. The peak reflectivity of this grating was $-3 \%$. (b) Bipolar grating reflectivity spectrum (theoretical and experimental). The refractive index phase superstructure is shown inset. The peak reflectivity of this grating was $-50 \%$.

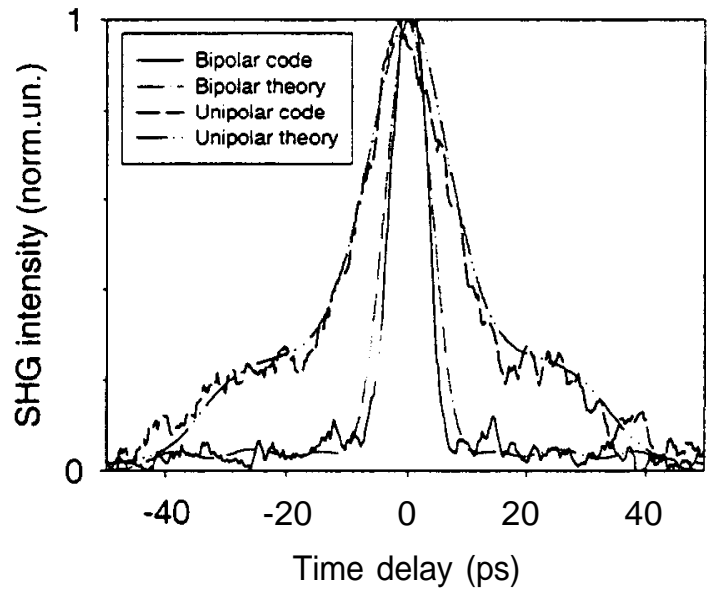

Fig.3 Theoretical and experimental pulse intensity autocorrelation functions for the code:decode process for both the unipolar and bipolar grating pairs.

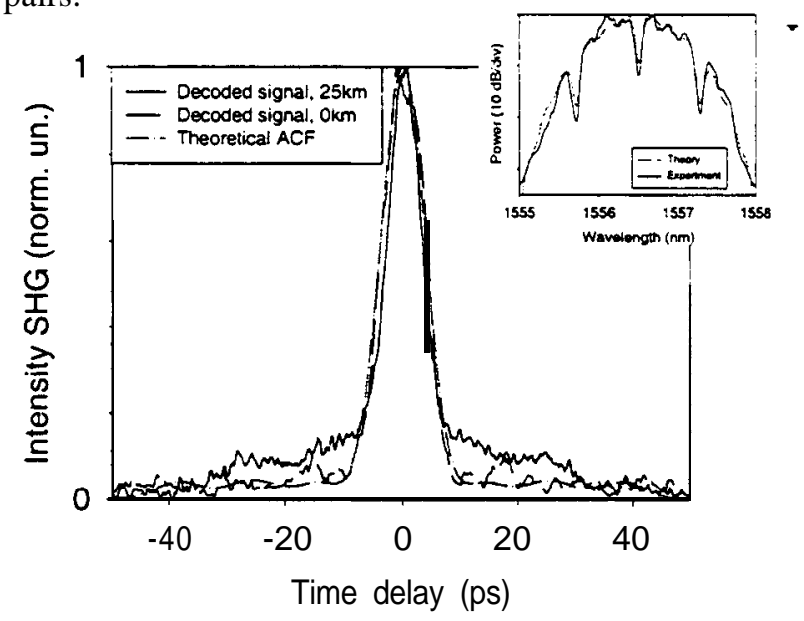

Fig 4 Theoretical and experimental pulse intensity autocorrelation functions for the code:decode process both before and after transmission through $25 \mathrm{~km}$ of (dispersion compensated) standard fibre.

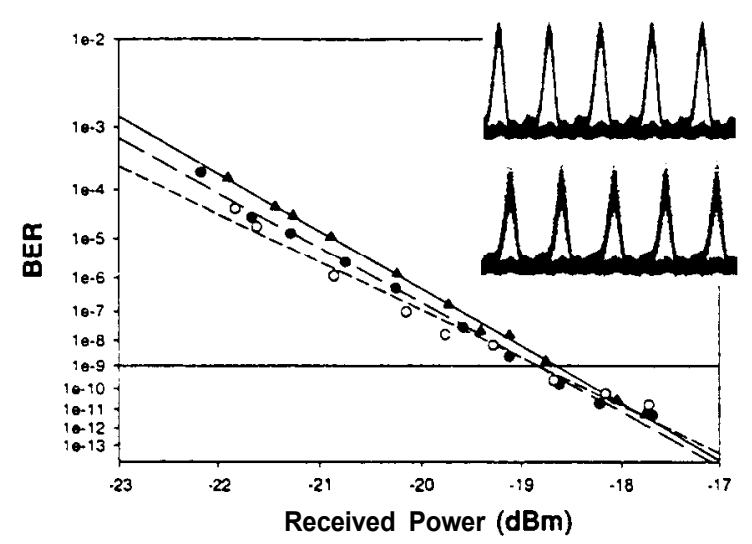

Fig.5 BER curves for back-to-back (open circles), and decoded signal before (closed circles) and after (triangles) transmission; the corresponding eye diagrams are shown inset 